\title{
Soleils nocturnes. Visions progressives dans les édifices à coupole de l'Antiquité tardive
}

\section{Vladimir Ivanovici}

\section{(2) OpenEdition}

\section{$\checkmark$ Journals}

Édition électronique

URL : http://journals.openedition.org/edl/1142

DOI : $10.4000 /$ edl. 1142

ISSN : 2296-5084

Éditeur

Université de Lausanne

\section{Édition imprimée}

Date de publication : 15 mai 2018

Pagination : 105-124

ISBN : 978-2-940331-68-0

ISSN : 0014-2026

Référence électronique

Vladimir Ivanovici, «Soleils nocturnes. Visions progressives dans les édifices à coupole de l'Antiquité tardive », Études de lettres [En ligne], 2 | 2018, mis en ligne le 15 mai 2020, consulté le 16 décembre 2020. URL : http://journals.openedition.org/edl/1142 ; DOI : https://doi.org/10.4000/edl.1142 


\section{SOLEILS NOCTURNES \\ VISIONS PROGRESSIVES DANS LES ÉDIFICES À COUPOLE DE L'ANTIQUITÉ TARDIVE}

Tenant compte du cadre nocturne du rituel baptismal tardo-antique, les décorations des coupoles des baptistères du $\mathrm{V}^{\mathrm{e}}$ siècle de Naples et de Ravenne dévoilent leurs secrets. C'est dans le contraste entre le fond bleu, qui se dématérialise dans l'obscurité, et les éléments réalisés avec le verre doré qu'un soleil nocturne émerge, constituant le point focal de la composition. La signification du motif, son caractère et sa fonction sont abordés dans cet article, jetant une lumière sur la dynamique complexe entre l'Église, les candidats et le cadre du baptême.

Et que dire du fait qu'ils cachent l'Invaincu (Sol Invictus) dans de sombres cavernes, et osent affirmer que celui qu'ils couvrent de ténèbres est le Soleil ? Qui pourrait honorer la lumière dans l'obscurité et cacher un astre céleste dans les enfers, si ce n'est par des pratiques mauvaises? ${ }^{1}$

Autour de la moitié du $\mathrm{V}^{\mathrm{e}}$ siècle, la réponse à cette question rhétorique sur ceux qui osaient enfermer le soleil dans une caverne pour l'adorer aurait profondément dérangé le poète chrétien. En effet, si l'on considère le cadre nocturne du baptême chrétien, les programmes décoratifs des baptistères $\mathrm{du} \mathrm{V}^{\mathrm{e}}$ siècle de Naples et de Ravenne deviennent témoins d'une mise en scène rituelle orchestrée par des évêques, cherchant à reproduire celle questionnée par le pseudo-Paulin. Les sources littéraires et archéologiques témoignent de la centralité du soleil nocturne dans les

I. Ps.-Paulinus (env. 380), Carmina, 32.113-116. 
rituels initiatiques des cultes d'Isis et de Mithra, ainsi que dans la théurgie néoplatonicienne: il s'agissait d'un motif de grande puissance. En raison de la nature de l'initiation chrétienne, destinée à ceux qui possédaient le vocabulaire symbolique de la société tardo-antique, mais pas encore celui du culte chrétien naissant, l'évêque employait un tel motif pour conférer crédibilité à l'initiation ${ }^{2}$. Mettant à profit la diffusion récente de l'emploi des mosaïques en pâtes de verre dans les coupoles, les évêques transformaient l'apparition des soleils nocturnes en une vision impressionnante, qui servait de base visuelle à une expérience qui changeait la vie ${ }^{3}$. La manière d'atteindre cet effet et sa signification dans le contexte de l'initiation seront présentés dans cette contribution, qui discute des conditions de vision, du public prévu et du but du rituel à travers les programmes décoratifs des espaces baptismaux tardo-antiques. Une telle lecture ouvre la voie à un nouvel horizon interprétatif des thèmes liés au baptême, tout en attirant l'attention sur la double dynamique dans laquelle les éléments liés au processus de catéchèse doivent être abordés.

Dans le récit le plus célèbre sur l'initiation antique, conservé dans le onzième livre des Métamorphoses d'Apulée (env. 170 apr. J.-C.), un soleil nocturne est montré à l'initié:

J’ai approché des limites de la mort; j’ai foulé le seuil de Proserpine, et j'en suis revenu porté à travers tous les éléments; en pleine nuit, j’ai vu le soleil briller d'une lumière étincelante; j'ai approché les dieux d'en bas et les dieux d'en haut; je les ai vus face à face et les ai adorés de près ${ }^{4}$.

En faisant appel à une vision du monde spécifique dans laquelle les niveaux cosmiques et célestes se chevauchaient, les cultes de l'Antiquité tardive présentaient l'apothéose de l'individu (vivant ou décédé) comme une ascension vers les parties supérieures du cosmos. Séparés par la constellation du Zodiaque, les espaces dédiés aux mortels et aux

2. En même temps, il semblerait que les rabbins sanctionnaient la représentation du Soleil dans les synagogues, cf. infra.

3. Sur la dynamique entre théologie, rituel et programmes décoratifs, cf. G. Hellemo, Adventus Domini.

4. Apulée, Les Métamorphoses, XI.23, p. 160. 
immortels étaient connectés par des chaînes ouvertes par la divinité 5 . En descendant et en montant, ces divinités offraient à leurs adeptes la possibilité de rejoindre les dieux immortels. Alors que la constellation du zodiaque marquait un seuil et fonctionnait en tant que symbole de ces vortex qui s'ouvraient à travers le pouvoir des rituels, le soleil était considéré comme un moteur de l'ascension. Cœur du cosmos, le soleil était le seul élément de la région supérieure visible depuis la Terre, instrumental en ce sens, car il assurait la traction nécessaire pour l'ascension ${ }^{6}$ (fig. 1). Anthropomorphisé, le culte du Soleil prit de l'élan au II ${ }^{\mathrm{e}}$ siècle, quand la théorie des cieux multiples fusionna avec la cosmologie ptoléméenne et rendit le cosmos visible en tant que part d'un système ontologique désormais unifié, qui plaçait les hommes à une extrémité et les dieux à l'autre ${ }^{7}$. Encouragé par les empereurs, qui promouvaient Sol comme un symbole de pouvoir impérial, le culte du Soleil se développa. Pour l'image de Sol, la religion traditionnelle emprunta des attributs à Jupiter/ Zeus, au point de remplacer ceux-ci au sommet du Panthéon, tandis que d'autres cultes incluaient Sol de différentes manières. En partageant la structure sous-jacente du monde, conçu sous la forme d'une succession de niveaux cosmiques et ontologiques, les cultes tardo-antiques attribuaient au Soleil un rôle inévitable dans leur imagerie cultuelle ${ }^{8}$. Des traits de Sol ont alors été transférés à d'autres divinités, représentées à sa manière, entourées d'autres divinités ou personnages secondaires. Comme l'a montré Jacques Flamant, "le choix d'une structure obéissant à un mouvement circulaire s'accorde avec la représentation de corps célestes - étoiles prises isolément, constellations, planètes - car cette structure reproduit la conception idéale d'un cosmos parfait, donc sphérique et ordonné, et fini. La régularité de ce type de structure, quelles

5. Selon B. Kühnel, "The synagogue floor mosaic in Sepphoris», p. 39, avant le $\mathrm{IV}^{\mathrm{e}}$ siècle «la constellation du zodiaque s'émancipa (dans l'art romain) de la configuration planétaire et devint la représentation par excellence du cosmos, ainsi qu'un symbole eschatologique" (trad. libre).

6. Cf. Proclus de Lycie (412-485), Hymne au soleil, 6, p. 149.

7. Le système dichotomique qui, dans l'Antiquité, opposait les mortels et les immortels avait été remplacé par un système dans lequel les deux catégories ontologiques étaient deux extrémités différentes d'une même chaîne de l'être.

8. Voir p. ex. la place attribuée à Sol dans le culte de Mithra et son rôle dans la formation de l'iconographie du Christ. 
qu'en soient les variantes, et sa parfaite symétrie par rapport au point central, expriment la loi qui régit tous les mouvements des êtres divins»?.

La popularité des initiations pendant l'Antiquité tardive, qui mettaient en scène des rituels d'entrée présentés comme des expériences bouleversantes et visionnaires, découle de l'adaptation des techniques employées dans les initiations traditionnelles, comme celles d'Éleusis ou de Samothrace ${ }^{10}$. En popularisant un type d'expérience réservée auparavant à une tranche étroite de la population, les cultes tardo-antiques s'appuyaient sur des cadres moins spectaculaires. L'apparition lumineuse de Déméter dans le Télestérion se transforma dans l'Antiquité tardive en celle de la principale divinité du culte, portant les attributs de Sol, ou de Sol lui-même. Les visions étaient d'habitude mises en scènes comme une combinaison d'obscurité, de lumière et de liquide; le reflet de la lumière dans le liquide était présenté comme une théophanie. L'évêque Hippolyte de Rome (170-235) précise de quelle manière se produisaient ces effets:

Et ils firent apparaître la lune et le soleil sur la voûte de cette manière. Dans la partie centrale de la voûte, ayant accroché un miroir, en plaçant un plat plein d'eau dans la portion centrale du pavement, de même qu'en mettant dans un endroit central un cierge, qui émettait une lumière pâle d'une position plus haute que le plat, de cette manière, à travers ce reflet, [le magicien] fait en sorte que la lune apparaît auprès du miroir ${ }^{11}$.

Ces techniques avaient été employées depuis l'Antiquité et furent adoptées et adaptées par les cultes tardo-antiques pour leurs besoins conceptuels et rituels ${ }^{12}$. Le culte de Mithra, qui fleurissait dans sa forme romaine aux $\mathrm{II}^{\mathrm{e}}$ et $\mathrm{III}^{\mathrm{e}}$ siècles, se fondait sur des artifices analogues. Les voûtes des grottes mithriaques étaient souvent parées d'écailles de poisson, dont le phosphore émettait une lumière terne, ou par des tessons de verre coloré, qui offraient des surfaces de réflexion aux lumières

9. J. Flamant, Macrobe et le néo-platonisme latin, à la fin du IVe siècle, p. 414.

IO. Sur ces phasmata dans l'Antiquité, voir J. N. Bremmer, Initiation into the Mysteries of the Ancient World, p. 68, avec la bibliographie.

II. Hippolyte de Rome, Contre les Hérésies, IV.37, p. 124 (trad. libre).

I2. Voir la fresque du I ${ }^{\text {er }}$ s. apr. J.-C. de la Villa des Mystères de Pompéi, où l'une des scènes montre de quelle manière la vision d'un satyre était créée à travers le reflet d'un masque dans un bol. 


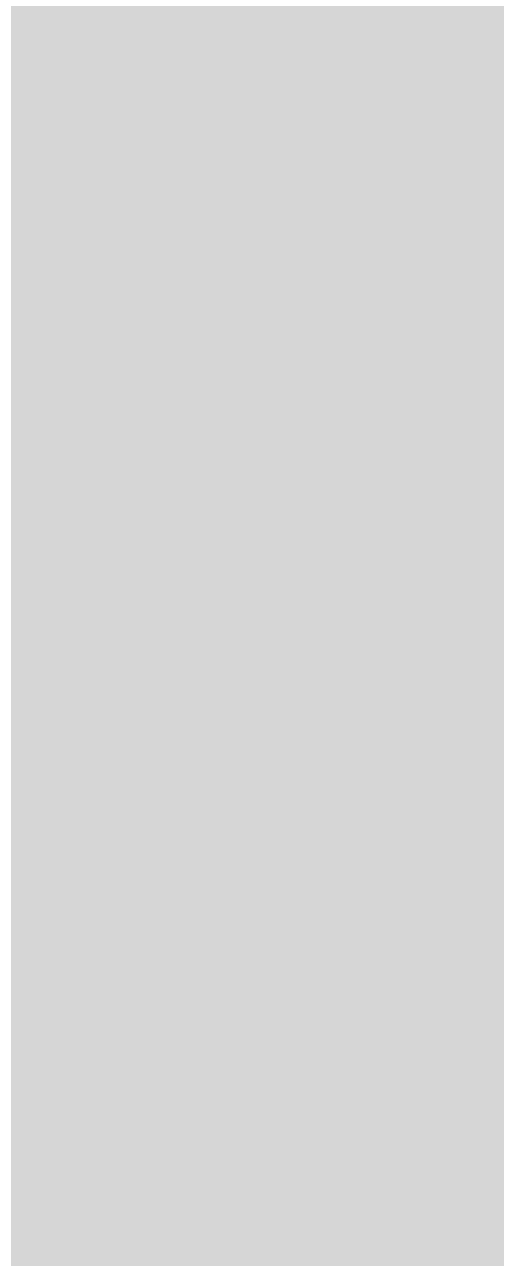

Fig. 1 - Londres, British Museum, Début du Ve siècle. Volet d'un diptyque en ivoire qui illustre l'apothéose de Quintus Aurelius Symmachus (env. 340-402). Deux victoires cornues soulèvent l'esprit de l'orateur au-delà de la constellation du zodiaque, où Sol l'attend; en même temps d'autres divinités planétaires regardent la scène au-dessus de la constellation.

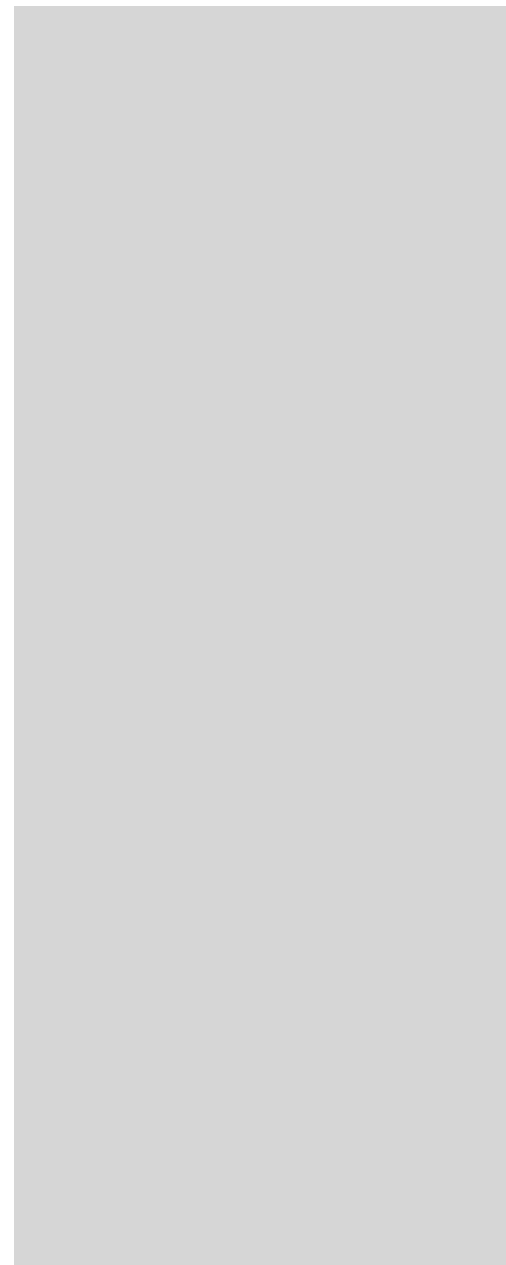

Fig. 2 - Carrawburgh. Autel de Sol avec des rayons creusés pour la projection de la lumière d'une lampe placée à l'intérieur de la stèle. Provenant du mithraeum de Brocolitia (III ${ }^{\mathrm{e}}$ siècle). 
employées dans l'espace du rituel ${ }^{13}$. Les images de Mithra et de Sol, les deux figures principales du culte, étaient décorées de feuilles d'or et présentaient souvent des niches cachées pour des lampes, qui les rendaient spectaculaires (fig. 2).

La phase tardo-antique du platonisme a vu le développement d'une pratique rituelle qui combinait les principes philosophiques avec les croyances cosmologiques de l'époque. La part de la pratique théurgique qui comportait la déification du praticien appelait à une théophanie dont la nature trouve des similarités avec l'imagerie isiaque, mithriaque et chrétienne ${ }^{14}$. Le symbole ou l'image du dieu étaient conçus pour apparaître sur une surface comme un spectacle lumineux. Une version anthropomorphisée de cette pratique, de grande importance en raison de ses similarités avec l'imagerie chrétienne successive, est décrite en détail dans l'ainsi-dite Liturgie de Mithra ${ }^{15}$. Le texte promettait à l'initié la vision d'êtres immortels, qui seraient devenus visibles «à travers le disque de dieu, mon père ${ }^{16}$. Comme le disque du soleil s'ouvrait, appelé par l'initié, il devenait un vortex à travers lequel la partie la plus haute du cosmos apparaissait. L’expérience atteignait bientôt son sommet: «les rayons du soleil se replient sur toi; regarde dans le centre de ceux-ci. Après, quand tu auras fait cela, tu verras un dieu jeune, d'une belle apparence, avec des cheveux enflammés, habillé d'une tunique blanche avec une cape écarlate et portant une couronne enflammée» ${ }^{17}$. L'adoption de l'imagerie solaire de la part du néoplatonisme, de même que sa représentation dans les synagogues à ce moment, confirme la grande importance de ce motif pour le public tardo-antique ${ }^{18}$.

I3. Sur les différents effets de la lumière, cf. V. Ivanovici, Manipulating theophany, p. 30-32, avec la bibliographie précédente.

I4. G. Shaw, Theurgy and the Soul, p. 171.

I5. S. I. Johnston, "Rising to the occasion", p. 183, n. 54, qui identifie la Liturgie de Mithra comme un texte théurgique.

I6. Liturgie de Mithra 548-549, p. 54-56 (trad. libre). Le disque de soleil est mentionné encore aux vers 551, 577, 581 et 582 .

17. Liturgie de Mithra 634-638, p. 54-56 (trad. libre).

I8. Le motif radial montrant le soleil personnifié ou stylisé conduisant son chariot à travers le centre du Zodiaque est représenté dans les synagogues de Sepphoris, Beth Alpha, Hammat Tiberias, Husifah et Na'aran. Sur le sens de ces mosaïques, cf. R. Hachlili, "The zodiac in ancient Jewish synagogal art»; J. Magness, "Heaven on Earth». 
Fig. 3 - Naples, Baptistère de San Giovanni in Fonte (début du Ve siècle). Mise en évidence des tesselles de mosaïque bleu foncées (en noir) et dorées (en gris) de la décoration de la coupole.

Au siècle suivant, la même vision était offerte à ceux qui se joignaient au culte du Christ à Ravenne. Dans le Baptistère des Orthodoxes, ou Néonien (autour de 458), l'image de Jésus était représentée dans la coupole au centre d'un motif solaire. La même mise en scène, bien qu'avec un effet encore plus direct, se trouve au Baptistère de San Giovanni in Fonte à Naples, qui précède de quelques décennies celui de Ravenne. À Naples, le motif solaire au centre fonctionnait, à la manière de la Liturgie de Mithra et des Métamorphoses d'Apulée, comme un oculus qui s'ouvrait sur le ciel nocturne, permettant à l'initié de voir le symbole de Dieu (fig. 3). À Ravenne, un effet encore plus sophistiqué était recherché. Comme remarqué avec perspicacité par Annabel Wharton, la décoration 
Fig. 4 - Ravenne, Baptistère des Orthodoxes (env. 458). Mise en évidence des tesselles de mosaïque bleu foncées (en noir) et dorées (en gris) de la décoration de la coupole.

du Baptistère Néonien montrait une «modification du vocabulaire traditionnel des formes: des dispositifs développés dans l'Antiquité pour créer l'illusion d'une réalité qui allait au-delà du plan de la peinture, commencèrent à être employés comme des moyens pour projeter l'image dans l'espace occupé par le public ${ }^{19}$. Au lieu de permettre au regard de l'initié de monter, ce cadre matérialisait la présence de Jésus et des apôtres dans l'espace même du baptistère. Cette mise en scène répondait à une théologie baptismale complexe et était basée sur une expérience rituelle qui faisait appel à la connaissance d'autres cultes pour offrir la vision de

19. A. J. Wharton, «Ritual and reconstructed meaning», p. 358 (trad. libre); cf. aussi S. K. Kostof, The Orthodox Baptistery of Ravenna, p. 168 sq. 
Dieu (fig. 4). C'était seulement une fois que les conditions objectives étaient en place, à savoir le cadre (nocturne, misant donc sur un éclairage artificiel), le public prévu (catéchumènes, dont le vocabulaire visuel et symbolique était celui de la société tardo-antique) et le but du rituel (la transformation du candidat dans un alter Christus à travers la vision du Christ pendant le rituel), que le motif du soleil nocturne, confondu avec une roue dans l'historiographie moderne, se révélait, en faisant lumière sur le caractère liminal de l'imagerie baptismale chrétienne ${ }^{20}$.

En revenant à la période qui suivit les dispositions de l'empereur Constantin (306-337), l'Église adopta des tendances et des motifs considérés efficaces dans la société tardo-antique. Comme l'a indiqué Dominic Janes dans son étude du phénomène, «cela permettait à la nouvelle secte de communiquer efficacement et d'entraîner un maximum de conversions " ${ }^{21}$. Dans le cas du baptême, ce processus commença plus tôt, quand une tradition apocryphe qui mentionnait une théophanie de lumière au moment du baptême de Jésus dans le Jourdain était reproduite de plusieurs façons lors de l'initiation. En raison de l'utilisation répandue des effets de lumière pendant d'autres rituels initiatiques, les communautés chrétiennes qui souhaitaient l'observance du même public tardo-antique mirent en scène l'apparition lumineuse ${ }^{22}$. Avec l'arrivée au rang d'évêque de figures conscientes à la fois des différentes façons employées depuis l'Antiquité pour produire des expériences changeant la vie, ainsi que de l'efficacité de ces artifices, une nouvelle mise en scène fut conçue pour le baptême. L'initiation a ainsi été reconceptualisée en tant que mort rituelle et son sujet était transformé par le baptême, miroitant la mort et la résurrection du Christ ${ }^{23}$. Présenté comme une expérience unique et à caractère transformant, à travers laquelle les bases ontologiques de chacun étaient changées, le baptême a été conçu sur la base des expériences faites par les autres cultes, aussi bien dans sa préparation que dans son organisation.

20. Sur la roue, cf. S. K. Kostof, The Orthodox Baptistery of Ravenna, p. 121; C. Muscolino, «Gli apparati decorativi», p. 37.

2I. D. Janes, God and gold in Late Antiquity, p. 45 (trad. libre).

22. Cf. A. Cosentino, "Il fuoco sul Giordano, il cero pasquale e la columna del Battistero Lateranense" sur la compétition et les différentes façons d'employer la lumière dans le rite.

23. Cf. p. ex. Jean Chrysostome, Bapt. Intr., 2.1., PG 49.233B. 
Une partie du processus consistait dans la mise en œuvre de tabous épistémiques, visuels et spatiaux, qui s'estompaient avec un effet cathartique à la fin du cycle ${ }^{24}$. Durant la préparation de l'expérience, des allusions étaient employées afin de créer une attente, qui était satisfaite pendant le rituel, toujours avec un effet cathartique. L'image du soleil nocturne faisait notamment partie de ce processus, lors duquel l'évêque se référait souvent à la dimension solaire de la vision promise, ainsi qu'à son effet sur les néophytes:

Rappelle-toi de moi, quand tu entreras dans ce royaume, quand tu recevras la robe royale, quand tu seras habillé de la pourpre, immergé dans le sang du Maître, quand tu poseras sur ta tête le diadème, dont le lustre se déploie de chaque côté avec une luminosité qui rivalise avec les rayons du soleil ${ }^{25}$.

En tant que symbole de la croyance dans l'ascension et dans l'au-delà, le soleil était représenté dans les catacombes aussi bien sous forme anthropomorphe que de manière stylisée. La composition que l'on trouve dans les coupoles des baptistères du $\mathrm{V}^{\mathrm{e}}$ siècle semble dépendre de l'art des catacombes, tant pour la combinaison du schéma radial développé dans la décoration des voûtes avec des images de Sol, que pour l'effet spécial produit par le verre doré. En utilisant la même technique employée pour les mosaïques dorées, les vaisselles avec des fonds de verre décorés à la feuille d'or étaient recyclées dans les parois des catacombes pour signaler, grâce à leurs iconographies, la foi ou l'identité du défunt (fig. 5). Dans les conditions de faible éclairage des catacombes chrétiennes, on peut observer l'effet particulier que les mosaïques en verre doré produisaient grâce à l'éclairage dynamique apporté par lampes et bougies. Rendu nocturne au début du $\mathrm{IV}^{\mathrm{e}}$ siècle afin d'augmenter justement l'effet des lumières et de signifier la théophanie, le baptême offrait un cadre

24. Cf. K. Clinton, "Stages of initiation in the Eleusinian and Samothracian Mysteries ", avec Cyril de Jérusalem, Procatechesis, 1.1, PG 33.332-3 et spécialement Ambroise de Milan, De mysteriis, 1.1.1, p. 136 sq.

25. Jean Chrysostome, Bapt. Instr. 1.1, p. 132 (trad. libre). Cf. aussi la formulation choisie par Basile le Grand, Traité du Saint Esprit, 9.23, p. 65: "Et lui, comme un soleil se saisissant d'un œil purifié, te montrera en lui-même l'Image de l'Invisible. Alors, dans la bienheureuse contemplation de l'Image, tu verras l'indicible beauté de l'Archétype». 
Fig. 5 - Parme, Museo Archeologico Nazionale, IVe-Ve siècle. Verre doré avec le Christ et les douze apôtres.

analogue ${ }^{26}$. Comme pour d'autres aspects ayant trait à la monumentalisation du rituel chrétien, les développements d'un motif de soleil nocturne pour la décoration des coupoles semblent remonter à l'époque de l'empereur Constantin. Dans une structure circulaire qu'il fonda sur la via Nomentana à Rome, près des catacombes qui abritaient le tombeau d'Agnès (l'ainsi dit Mausolée de Santa Costanza), la coupole était décorée avec des mosaïques qui présentaient le même dispositif que celles de Naples et de Ravenne: sur un fond bleu foncé, un motif solaire central était rendu par des tesselles dorées et d'autres scènes étaient représentées 
dans l'apparente profondeur suggérée par le contraste entre les deux couleurs $^{27}$.

À Naples, au tournant du siècle, la même technique est adaptée pour reproduire la scène décrite dans les Métamorphoses d'Apulée et dans la Liturgie de Mithra. En jouant sur l'attente de la part du public de trouver un vrai oculus, que les édifices circulaires de ce type avaient souvent, le concepteur a fait en sorte qu'un soleil à huit rayons constitué de tesselles dorées apparaisse sur ce qui semblait être un ciel nocturne - un fond bleu foncé dématérialisé dans la nuit ${ }^{28}$. Comme dans la Liturgie de Mithra, le disque de soleil s'ouvrait pour permettre la vision du symbole $\mathrm{du}$ Christ, motif solaire en soi $^{29}$. Cela était rendu possible par l'abondant éclairage artificiel, caractéristique de l'initiation, qui dirigeait l'attention sur les parties décorées avec l'or ${ }^{30}$. En reproduisant le concept du baptême comme une ouverture des cieux par l'Église pour ses néophytes, formulé par l'évêque Maxime de Turin († 420 env.), cette iconographie supporte notre lecture de la mise en scène comme une interprétation christianisée d'une expérience visuelle commune à l'époque:

On doit donc ouvrir le ciel à nos candidats, car pour eux il est encore fermé. Il est encore fermé pour eux, car ils ne connaissent pas le mystère de la Trinité. En fait, le ciel étant pour eux encore fermé, ils ignorent ce qui arrive en dessus de celui-ci, et ils ne peuvent pas connaître quelle est la nature du Fils et du Père, avant d'aller au-delà des éléments du monde. Un pourra contempler les mystères divins de la Trinité quand grâce à sa vertu il aura les cieux ouverts, comme le bienheureux martyr Étienne s'ouvrit les cieux avec son martyre et vit le Sauveur qui se tenait à la droite du Père qui lui dit: celui auquel

27. La décoration est connue grâce à des dessins du XVIII e siècle, cf. M. Andaloro (ed.), L'orizzonte tardoantico e le nuove immagini (312-468), p. 76.

28. La crédibilité de la vision était assurée par la tension physiologique et psychologique placée sur les catéchumènes à travers le jeûne, l'obscurité, la privation de sommeil et la peur - des aspects attestés dans le processus de catéchèse et connus pour leur capacité de modifier la perception. Cf. J. J. Pilch, "Holy men and their sky journeys", p. 108 sq.; V. Ivanovici, Manipulating theophany, p. 20 sq.

29. Sur le chi-rho inscrit dans un cercle comme symbole solaire cf. M. Wallraff, Christus verus Sol, p. 154 sq.

30. Le fait que le baptême était désigné «illumination» et le baptistère «lieu de l'illumination", ainsi que sa représentation comme un sacrement caractérisé par le feu, la lumière et des dispositifs d'illumination atteste la centralité de la lumière dans ce domaine. Cf. V. Ivanovici, Manipulating theophany, p. 25 sq. 
Fig. 6 - Aquileia, Museo Nazionale Paleocristiano, $\mathrm{V}^{\mathrm{e}}$ siècle. Scène de baptême incisée sur une dalle funéraire: le font correspond à un oculus qui s'ouvre et permet au ciel nocturne, aux eaux et à la colombe d'apparaître.

les cieux sont fermés doit œuvrer afin qu'ils s'ouvrent, afin de voir le Christ au-dessus des cieux. En effet, jusqu'à ce qu'ils restent fermés pour un homme, il ne peut pas voir le Christ qui règne ${ }^{31}$. (fig. 6)

À Ravenne, le programme est plus élaboré. Le soleil est construit soigneusement, avec le disque central représentant le baptême dans le Jourdain, les douze apôtres habillés en or, ainsi que douze candélabres dorés faisant office de rayons émanés par le Christ ${ }^{32}$. Modifié

31. Maxime de Turin, Sermons, 52.3, p. 211 (trad. libre).

32. Outre l'identification en tant que rayons de soleil dispersant la lumière du Christ, conformément à Mt. 5.14, la relation est aussi évidente avec les douze signes du zodiaque. L'association des apôtres avec les signes et les mois remonte déjà au II e siècle et fait partie d'une tradition qui voyait l'Incarnation comme un événement cosmique ayant pour but la libération des âmes du désordre du monde sublunaire. Sur la question, cf. T. Hegedus, Early Christianity and ancient astrology, p. 167 sq. et J. Daniélou, "Les douze Apôtres et le zodiaque». Comme dans la scène similaire représentée dans la synagogue de Sepphoris, où chaque signe est accompagné par une figure masculine 
actuellement par le rehaussement du niveau du pavement et par la perte de teinte des tesselles bleues de la mosaïque du fond, le motif solaire devait apparaître de manière évidente et immédiate aux candidats à cause de sa récurrence dans les initiations et des nombreuses allusions faites pendant la catéchèse.

Dans le Baptistère des Orthodoxes, les candidats se trouvaient face à une image spéculaire, suggérant que c'était notamment le Christ qui accomplissait le rituel auquel ils étaient en train de se soumettre. La mise en scène avait donc des implications profondes, car elle contribuait à remodeler le néophyte à l'image de son Dieu. La lumière du soleil nocturne ne se répandait pas seulement à l'intérieur du baptistère, mais aussi sur l'eau de la piscine, transformant le candidat en image vivante du Christ :

C'est pourquoi, mes frères, l'Ésprit céleste, avec son effusion mystérieuse de lumière, féconde l'utérus virginal du font, pour donner naissance à des créatures divines et apporter à la ressemblance au Créateur ceux qui, étant d'une race d'argile, avaient été jetés sur terre dans une condition misérable. Né à nouveau, reformé à l'image de notre Créateur, on accomplit ce que l'apôtre avait conseillé: comme on est né avec l'image de l'homme terrestre, laisse-nous porter aussi l'image de l'homme céleste ${ }^{33}$.

Une fois que les corps oints et mouillés des candidats reflétaient les lumières à l'intérieur du baptistère, l'évêque prétendait que la personne

flanquée par une étoile (les patriarches), le concepteur du baptistère a donné une expression visuelle à la prétention du culte de libérer les membres du caractère arbitraire de la rota nativitatis en replaçant les signes avec les apôtres.

33. Pierre Chrysologue, Sermons, 117.4-5, p. 376 (trad. libre). Pierre Chrysologue (env. 380-450) était le principal théologien de Ravenne. Le programme du Baptistère des Orthodoxes, réalisé quelques années après sa mort, reflète son interprétation christomorphe du rite. Le Chrysologue reprenait une bonne partie de son imagerie de Zénon de Vérone (env. 300-371), qui a décrit à plusieurs reprises la relation entre le Christ et les apôtres comme une composition radiale avec Sol entouré par les signes du zodiaque (cf. Zénon de Vérone, Discours 2, 12.2, p. 284-287) et qui, en se plaignant de son baptistère pauvrement décoré par rapport à des temples précieusement embellis, disait: "celui dans lequel le soleil y est toujours ne doit pas prier pour la lumière des fenêtres" (Zénon de Vérone, Discours 2, 6.3, p. 256, trad. libre). 
regagnait la luminosité Adamique ${ }^{34}$. Compte tenu du fait que les candidats étaient appelés des christs (littéralement: les oints), le chrisme et l'éclairage permettaient la perception et l'autoperception du néophyte comme image du Christ ${ }^{35}$. Sous le soleil nocturne, ils ressortaient aussi du font baptismal, comme le Christ, en tant qu'incarnations du soleil:

[dans le jour de l'Incarnation] une Vierge ressembla au Ciel, quand des rayons de lumière sont sortis de son ventre, quand le Soleil incarné a pris naissance dans son sein, quand la lumière s'est revêtue de la figure humaine, quand la naissance n’a pas été le commencement, mais seulement l'apparition de celui qui venait au monde ${ }^{36}$.

Ladoption du motif du soleil nocturne nous renseigne sur la dynamique de l'expérience baptismale, modelée sur les attentes de son public. Cette stratégie a assuré le succès du processus de sacrement, tout en rendant les programmes décoratifs à l'intérieur des baptistères fondamentalement ambivalents, car ils reflétaient le caractère liminal propre à leur public. L'analyse des espaces et du rituel baptismaux doit donc être faite en conséquence, en relation non seulement avec les attentes partagées de l'individu dans l'Antiquité tardive, mais aussi avec les sermons prébaptismaux, qui façonnaient la perception de l'expérience rituelle.

Vladimir IvanovicI Masaryk University Brno, Department of the History of Art, Centre for Early Medieval Studies Università della Svizzera Italiana, Istituto di Storia e Teoria dell'Arte e dell'Architettura

34. L'idée est récurrente dans les hymnes d'Ephrem le Syriaque (env. 306-373) et dans d'autres textes liés au baptême.

35. Cf. V. Ivanovici, Manipulating theophany, p. 46-48.

36. Proclus de Constantinople, Homélies, 2.10.149, p. 352 (trad. libre). 


\section{BIBLIOGRAPHIE}

\section{Sources}

Ambroise de Milan, De sacramentis, De mysteriis, Explanatio symboliDes sacrements. Des mystères, Explication du symbole, éd. par Dom Bernard Botte, Paris, Cerf, 1994 (2 eréimpression de la nouvelle édition).

Apulée, Les Métamorphoses, éd. par Donald S. Robertson, trad. par Paul Valette, vol. 3, Paris, Les Belles Lettres, 1971.

Basile le Grand, Traité du Saint Esprit, trad. par Annette Maignan, Paris, Desclée, 1979.

Cyril de Jérusalem, The Works of Saint Cyril of Jerusalem, vol. 1, trans. by Leo P. McCauley, Anthony A. Stephenson, Washington D.C., Catholic University of America Press, 1969.

Hippolyte de Rome, Contre les Hérésies - Hippolytus. Refutatio Omnium Haeresium, hrsg. von Miroslav Marcovich, Berlin, Walter de

Gruyter, 1986; trad. par John Henry Macmahon, Hippolytus (Pseudo). The Refutation of All Heresies, in Ante-Nicene Fathers. Vol. 5: Third-century Fathers, ed. by Alexander Roberts, James Donaldson, New York, Cosimo, [1886] 2007.

Jean Chrysostome, Bapt. Instr. - John Chrysostom. Baptismal Instructions, trans. by Paul W. Harkins, Westminster/London, Newman Press/Longmans Green, 1963.

Liturgie de Mithra - éd. et trad. par Hans Dieter Betz, The "Mithras Liturgy». Text, Translation and Commentary, Tübingen, Mohr Siebeck, 2003.

Maxime de Turin, Sermons - Maximus Taurinensis. Sermones, ed. by Almut Mutzenbecher, Turnholt, Brepols, 1962; trad. par Boniface Ramsey, The sermons of St. Maximus of Turin, New York, Newman Press, 1989.

PG - J.-P. Migne (éd.), Patrologiae cursus completus. Series Graeca, Paris, 1857-1866. 
Pierre Chrysologue, Sermons - Pietro Crisologo. Vol. 2: Sermoni 63-124, a cura di Gabriele Banterle et alii, Roma, Città Nuova Editrice, 1996.

Proclus de Constantinople, Homélies - Proclus of Constantinople and the cult of the Virgin in Late Antiquity. Homilies 1-5, trans. by N. P. Constas, Leiden/Boston, Brill, 2003.

Proclus de Lycie, Hymne au soleil - Proclus' Hymns: essays, translations, commentary, ed. by R. M. van den Berg, Leiden/Boston, Brill, 2001.

Ps.-Paulinus, Carmina - «Ps.-Paulinus Poema ultimum: texte et traduction", éd. et trad. par Anne-Marie Boxus et Jacques Poucet, Folia Electronica Classica, 20 (2010), en ligne <http://bcs.fltr.ucl. ac.be/FE/20/Poema/Texte.htm> (14.11.2017).

ZÉnon de Vérone, Discours - San Zenone di Verona. I Discorsi, a cura di Gabriele Banterle, Milano/Roma, Biblioteca Ambrosiana/Città Nuova Editrice, 1987.

\section{Études}

Andaloro, Maria (ed.), L'orizzonte tardoantico e le nuove immagini (312-468), Milano, Jaca Book, 2006.

Bremmer, Jan N., Initiation into the Mysteries of the Ancient World, Berlin, Walter de Gruyter, 2014.

Clinton, Kevin, "Stages of initiation in the Eleusinian and Samothracian Mysteries", in Greek Mysteries: the archaeology and ritual of ancient Greek secret cults, ed. by Michael B. Cosmopoulos, London, Routledge, 2003, p. 50-78.

Cosentino, Augusto, "Il fuoco sul Giordano, il cero pasquale e la columna del Battistero Lateranense", in L'edificio battesimale in Italia. Aspetti e problemi. Atti dell'VIII congresso nazionale di archeologia cristiana (21-26.9.1998), Bordighera, Istituto Nazionale di Studi Liguri, 2001, p. 521-540.

Daniélou, Jean, "Les douze Apôtres et le zodiaque", Vigiliae Christianae, 13/1 (1959), p. 14-21.

Flamant, Jacques, Macrobe et le néo-platonisme latin, à la fin du IV siècle, Leiden, E. J. Brill, 1977. 
HachliLI, Rachel, "The zodiac in ancient Jewish synagogal art: a review", Jewish Studies Quarterly, 9 (2002), p. 219-258.

Hegedus, Tim, Early Christianity and ancient astrology, New York, Peter Lang, 2007.

Hellemo, Geir, Adventus Domini: eschatological thought in fourthcentury apses and catecheses, Leiden, E. J. Brill, 1989.

IvanovicI, Vladimir, Manipulating theophany. Light and ritual in North Adriatic architecture (ca. 400-ca. 800), Berlin, Walter de Gruyter, 2016.

Janes, Dominic, God and gold in Late Antiquity, Cambridge, Cambridge University Press, 1998.

Johnston, Sarah Illes, «Rising to the occasion: theurgic ascent in its cultural milieu", in Envisioning magic: a Princeton seminar and symposium, ed. by Peter Schaefer, Hans G. Kippenberg, Leiden, E. J. Brill, 1997, p. 165-194.

Kostof, Spiro K., The Orthodox Baptistery of Ravenna, New Haven/ London, Yale University Press, 1965.

KüHnel, Bianca, "The synagogue floor mosaic in Sepphoris: between Paganism and Christianity", in From Dura to Sepphoris. Studies in Jewish art and society in Late Antiquity, ed. by Lee I. Levine, Zeev Weiss, Journal of Roman Archaeology, (2000), p. 31-43.

Magness, Jodi, "Heaven on Earth: Helios and the zodiac cycle in ancient Palestinian synagogues ", Dumbarton Oaks Papers, 59 (2005), p. 1-52.

Muscolino, Cetty, "Gli apparati decorativi», in Il Battistero Neoniano: uno sguardo attraverso il restauro, a cura di Cetty Muscolino, Antonella Ranaldi, Claudia Tedeschi, Ravenna, Longo Editore, 2011, p. 33-54.

Pilch, John J., «Holy men and their sky journeys : a cross cultural model», Biblical Theology Bulletin, 35 (2005), p. 106-112.

SHaw, Gregory, Theurgy and the Soul: the Neoplatonism of Iamblichus, University Park, The Pennsylvania State University Press, 1995.

Wallraff, Martin, Christus verus Sol: Sonnenverehrung und Christentum in der Spätantike, Münster, Aschendorff, 2001. 
Wharton, Annabel J., "Ritual and reconstructed meaning: the Neonian Baptistry in Ravenna», The Art Bulletin, 69/3 (1987), p. $358-365$.

\section{Crédits iconographiques}

Fig. 1 : Dessin de D. Ivanovici.

Fig. 2: Merkelbach, Reinhold, Mithras. Ein persisch-römischer Mysterienkult, Wiesbaden, VMA-Verlag, 1998, p. 334.

Fig. 3: Dessin d'après Bertaux, Émile, L'art dans l'Italie méridionale. Vol. 1: De la fin de l'empire romain à la conquête de Charles d'Anjou, Paris, Albert Fontemoing, 1904, p. 47 (développement graphique de Lorenzo R. Pini).

Fig. 4: Dessin de Lorenzo Russo, développement graphique de Lorenzo R. Pini.

Fig. 5: <https:/www.numismaticadellostato.it/web/pns/patrimonio/ vetrine/parma/galleria-fotografica> (20.8.2017).

Fig. 6 : Jensen, Robin M., Living water: images, symbols, and settings of early Christian baptism, Leiden/Boston, Brill, 2010, p. 72. 
